\title{
Editorial
}

\section{Acknowledgement to Reviewers of the Journal of Clinical Medicine in 2013}

Journal of Clinical Medicine Editorial Office, MDPI AG, Klybeckstrasse 64, CH-4057 Basel, Switzerland

Published: 24 February 2014

The editors of the Journal of Clinical Medicine would like to express their sincere gratitude to the following reviewers for assessing manuscripts in 2013:

$\begin{array}{lll}\text { Almog, Nava } & \text { Drago, Denise } & \text { Hirai, Yuji } \\ \text { Amadori, Dino } & \text { Economou, Athina } & \text { Houssiau, Frederic A. } \\ \text { Antebi, Ben } & \text { Esteban, Andrés } & \text { Hubalek, Michael } \\ \text { Bernal-Mizrachi, Carlos } & \text { Evans, Mark } & \text { Ingvild, Ingvild } \\ \text { Bourin, P. } & \text { Facchini, Andrea } & \text { Josson, Sajni } \\ \text { Bouza, Emilio } & \text { Fong, Daniel Tik-Pui } & \text { Kasten, Mary J. } \\ \text { Bove, Geoffrey } & \text { Foroozan, Rod } & \text { Kim, Han Su } \\ \text { Bower, Chris } & \text { Frölich, J. } & \text { Kohn, Michael R. } \\ \text { Brodowicz, Thomas } & \text { Füllen, Georg } & \text { Kusuma, Sravanti } \\ \text { Broggini, Massimo } & \text { Galanter, Cathryn A. } & \text { Kwon, Tae Gyun } \\ \text { Brufsky, Adam } & \text { Goetzl, Laura } & \text { Languino, Lucia } \\ \text { Califaretti, Nadia } & \text { Goldstein, Benjamin I. } & \text { Laurie, Karen L. } \\ \text { Casimiro, Sandra } & \text { Gräs, Søren } & \text { Lee, Moon Soo } \\ \text { Cavoretto, Paolo } & \text { Gunetti, Monica } & \text { Li, Gang } \\ \text { Connelly, Kim } & \text { Hagmann, Sébastien } & \text { Lipton, Allan } \\ \text { Conover, Joanne } & \text { Halperin, John } & \text { Lu, Baisong } \\ \text { Cousens, Nicole } & \text { Harper, Gordon } & \text { Lungu, Codrin } \\ \text { Cunha, Burke A. } & \text { Hashmi, Farina } & \text { Luparello, Claudio } \\ \text { Dahlström, Lisen Arnheim } & \text { Haubitz, Sebastian } & \text { Lutz, Stephen } \\ \text { Davies, Robert D. } & \text { Hematti, Peiman } & \text { Magann, Everett F. } \\ \text { De Geest, Bart } & \text { Henry, David } & \text { Mccloskey, Eugene } \\ \text { Dennis, Kristopher } & \text { Hernigou, Philippe } & \text { Mlineritsch, Brigitte }\end{array}$


Montgomery, Scott

Morris, Patrick G.

Mortara, Franco

Narod, Steven

Ngim, Chin Fang

Nilar, Shahul

OBrien, Louise

Odejinmi, F.

Padfield, Gareth J.

Park, Won

Pergament, Eugene

Pergolizzi, Stefano

Polo, Jose

Pontikoglou, Charalampos

Porpora, M. G.

Powles, Trevor

Radisky, Derek

Reed, Elizabeth
Rincón, Olga Gómez del

Rodriguez, Anne-marie

Rosenblatt, Jon E.

Salgado, A. J.

Schul, Wouter

Schuleri, Karl

Selvaraj, Vimal

Sesti, Francesco

Shaak, Thomas L.

Sonne, Susan C.

Sprick, Martin R.

Springett, Kate

Sridharan, Shankar

Stoddard, Joel

Stoeger, Herbert

Subbulakshmi, Subbulakshmi

Sundar, Krishna M.

Sykes, Jane
Takebe, Naoko

Teich, Steven

Trial, Joann

Truong, Daniel

Vagefi, Reza

Van Poznak, Catherine

Verhagen, Ealm

Wang, Qun

Wang, Yun

Watanabe, Shigeru

Wilson, Kumanan

Yasuhara, Takao

Yennamalli, Ragothaman

$\mathrm{Yu}$, Seongjin

Zhang, Jian

Zhang, Yong

Zhu, Xuegong

(C) 2014 by the authors; licensee MDPI, Basel, Switzerland. This article is an open access article distributed under the terms and conditions of the Creative Commons Attribution license (http://creativecommons.org/licenses/by/3.0/). 